\title{
On partial geometries arising from maximal arcs
}

\author{
Mustafa Gezek ${ }^{1}$ and Vladimir D. Tonchev² \\ ${ }^{1}$ Department of Mathematics, Tekirdag Namik Kemal \\ University, Tekirdag, Turkey 59030, mgezek@nku.edu.tr \\ ${ }^{2}$ Department of Mathematical Sciences, Michigan \\ Technological University, Houghton, MI USA 49931, \\ tonchev@mtu.edu
}

September 1, 2020

\begin{abstract}
The subject of this paper are partial geometries $p g(s, t, \alpha)$ with parameters $s=d\left(d^{\prime}-1\right), t=d^{\prime}(d-1), \alpha=(d-1)\left(d^{\prime}-1\right), d, d^{\prime} \geq$ 2. In all known examples, $q=d d^{\prime}$ is a power of 2 and the partial geometry arises from a maximal arc of degree $d$ or $d^{\prime}$ in a projective plane of order $q$ via a known construction due to Thas [28] and Wallis [34], with a single known exception of a partial geometry $p g(4,6,3)$ found by Mathon [22] that is not associated with a maximal arc in the projective plane of order 8. A parallel class of lines is a set of pairwise disjoint lines that covers the point set. Two parallel classes are called orthogonal if they share exactly one line. An upper bound on the maximum number of pairwise orthogonal parallel classes in a partial geometry $G$ with parameters $p g\left(d\left(d^{\prime}-1\right), d^{\prime}(d-1),(d-\right.$ $\left.1)\left(d^{\prime}-1\right)\right)$ is proved, and it is shown that a necessary and sufficient condition for $G$ to arise from a maximal arc of degree $d$ or $d^{\prime}$ in a projective plane of order $q=d d^{\prime}$ is that both $G$ and its dual geometry contain sets of pairwise orthogonal parallel classes that meet the upper bound. An alternative construction of Mathon's partial geometry is presented, and the new necessary condition is used to demonstrate why this partial geometry is not associated with any maximal arc in the projective plane of order 8 . The partial geometries associated with
\end{abstract}

\footnotetext{
${ }^{*}$ Corresponding author, tonchev@mtu.edu
} 
all known maximal arcs in projective planes of order 16 are classified up to isomorphism, and their parallel classes of lines and the 2-rank of their incidence matrices are computed. Based on these results, some open problems and conjectures are formulated.

Keywords: partial geometry, projective plane, maximal arc, strongly regular graph.

\section{Introduction}

We assume familiarity with basic facts and notions from combinatorial design theory [3], 33].

A partial geometry with parameters $s, t, \alpha$, or shorty, $p g(s, t, \alpha)$, is a pair $(P, L)$ of a set $P$ of points and a set $L$ of lines, with an incidence relation between points and lines, satisfying the following axioms:

1. A pair of distinct points is not incident with more than one line.

2. Every line is incident with exactly $s+1$ points $(s \geq 1)$.

3. Every point is incident with exactly $t+1$ lines $(t \geq 1)$.

4. For every point $p$ not incident with a line $l$, there are exactly $\alpha$ lines $(\alpha \geq 1)$ which are incident with $p$, and also incident with some point incident with $l$.

In terms of the parameters $s, t, \alpha$, the number $v=|P|$ of points, and the number $b=|L|$ of lines of a partial geometry $\operatorname{pg}(s, t, \alpha)$ are given by eq. (1).

$$
v=\frac{(s+1)(s t+\alpha)}{\alpha}, b=\frac{(t+1)(s t+\alpha)}{\alpha} .
$$

If $G=(P, L)$ is a partial geometry $p g(s, t, \alpha)$, the incidence structure $G^{\prime}$ having as points the lines of $G$, and having as lines the points of $G$, where a point and a line are incident in $G^{\prime}$ if and only if the corresponding line and a point of $G$ are incident, is a partial geometry $p g(t, s, \alpha)$, called the dual of $G$.

Partial geometries were introduced by R. C. Bose [4]. In the original Bose's notation, the number $t+1$ of lines incident with a point is denoted by $r$, and the number $s+1$ of points incident with a line is denoted by $k$. The $(s, t, \alpha)$-notation was adopted later to match the notation for generalized quadrangles, which are partial geometries with $\alpha=1$ [25]. A partial geometry $\operatorname{pg}(s, t, \alpha)$ is called proper if $1<\alpha<\min (s, t)$. 
In this paper, we consider proper partial geometries with parameters

$$
s=q-d, t=q(d-1) / d, \alpha=(q-d)(d-1) / d,
$$

where $q>d \geq 2$ are integers and $d$ divides $q$. All known partial geometries with parameters (2) arise from maximal arcs of degree $d=2^{m}$ in projective planes of order $q=2^{h}, h \geq 2$, via a construction due to Thas [28, [29], [31, Construction 41.21], and Wallis [34, with a single exception for $h=3$ and $m=1$, when in addition to the partial geometry $p g(6,4,3)$ arising from a maximal arc of degree 2 , that is, a hyperoval in $P G(2,8)$, there exists a second nonisomorphic partial geometry with the same parameters that was found by Rudi Mathon [21], and is not associated with any hyperoval in $P G(2,8)$.

If $d=2^{m}$ and $q=2^{h},(m<h)$, the parameters (2) can be written as

$$
s=2^{h}-2^{m}, t=2^{h}-2^{h-m}, \alpha=\left(2^{m}-1\right)\left(2^{h-m}-1\right),(1 \leq m<h) .
$$

The parameters (3) correspond to partial geometries of Type 1 in the classification of the known proper partial geometries given in [31, Theorem 41.31]. (The only known "improper" partial geometry with parameters (3) is a generalized quadrangle $p g(2,2,1),(m=1, h=2)$, arising from a hyperoval in the projective plane of order 4 .)

In Section 2, we prove a necessary and sufficient condition for a partial geometry with parameters (2) to be associated with a maximal arc of degree $d$ in a projective plane of order $q$ in terms of parallel classes of lines (Theorem 2.4).

In Section 3, we give an alternative construction of Mathon's partial geometry with parameters $s=6, t=4, \alpha=3$, and use Theorem 2.4 to show why this partial geometry is not associated with a maximal arc in $P G(2,8)$.

In Section 4, we examine the partial geometries associated with maximal arcs in projective planes of even order $q \leq 16$. The partial geometries associated with all known maximal arcs in projective planes of order 16 are classified up to isomorphism, and their parallel classes of lines and the 2rank of their incidence matrices are computed. Based on the results of these computations, some open problems and two conjectures are formulated.

\section{Maximal arcs and partial geometries}

Let $k$ and $d$ be positive integers such that $k>d>1$. A $(k, d)$-arc (or an arc of size $k$ and degree $d$ ) in a projective plane $\mathcal{P}$ of order $q$ is a set $\mathcal{A}$ of $k$

\footnotetext{
${ }^{1}$ A maximal arc of degree $d$ in a projective plane of order $q=d d^{\prime}$ is a set $\mathcal{A}$ of $q d-q+d$ points such that every line is either disjoint from $\mathcal{A}$ or meets $\mathcal{A}$ in exactly $d$ points.
} 
points such that $d$ is the greatest number of collinear points in $\mathcal{A}$. It follows that $k \leq d q-q+d$, and the equality $k=d q-q+d$ holds if and only if every line is either disjoint from $\mathcal{A}$ or intersects $\mathcal{A}$ in exactly $d$ points. A $(d q-q+d, d)$-arc is called a maximal arc. A maximal arc of degree $d=2$ is called also a hyperoval.

A necessary condition for the existence of a maximal arc of degree $d$ in a projective plane of order $q$ is that $d$ divides $q$. If $\mathcal{A}$ is a maximal $(d q-q+d, d)$ arc in a projective plane $\mathcal{P}$ of order $q$, then the lines of $\mathcal{P}$ that are disjoint from $\mathcal{A}$ form a maximal $(q(q-d+1) / d, q / d)$-arc $\mathcal{A}^{\prime}$ in the dual plane $\mathcal{P}^{\prime}$, called the dual arc of $\mathcal{A}$.

Maximal arcs of degree $d, 1<d<q$, do not exist in any Desarguesian plane of odd order $q$ (Ball, Blokhuis, and Mazzocca [2]), as well as in any of the four projective planes of order 9 (Lunelli and Sce [20]), and are known to exist in every Desarguesian plane of order $q=2^{t}$ (Denniston [8], Hamilton and Mathon [14, [15], Mathon [22], Thas [29]), as well as in some nonDesarguesian planes of even order (Gezek, Mathon and Tonchev [9], Gezek, Tonchev and Wagner [10], Hamilton [11, [12, [13, Hamilton, Stoichev and Tonchev [16], Penttila, Royle, and Simpson [26], Thas [30]).

If $\mathcal{P}$ is a projective plane of order $q=d d^{\prime}$, then a maximal arc $\mathcal{A}$ in $\mathcal{P}$ of degree $d$ has $d\left(q-d^{\prime}+1\right)$ points, while its dual arc $\mathcal{A}^{\prime}$ is of degree $d^{\prime}$ and has $d^{\prime}(q-d+1)$ points. The set of points of a maximal $\left(d\left(q-d^{\prime}+1\right), d\right)$-arc $\mathcal{A}$ and the non-empty intersections of $\mathcal{A}$ with lines of $\mathcal{P}$ considered as blocks, form a Steiner $2-\left(d\left(q-d^{\prime}+1\right), d, 1\right)$ design $D$. We say that $D$ is associated with the maximal arc $\mathcal{A}$, or that $D$ is embeddable in $\mathcal{P}$ as a maximal arc. Similarly, the points of the dual arc $\mathcal{A}^{\prime}$ define a Steiner $2-\left(d^{\prime}(q-d+1), d^{\prime}, 1\right)$ design $D^{\prime}$ embeddable in the dual plane $\mathcal{P}^{\prime}$.

A necessary and sufficient condition for a Steiner $2-\left(d\left(q-d^{\prime}+1\right), d, 1\right)$ design $D$ to be embeddable as a maximal $\left(d\left(q-d^{\prime}+1\right), d\right)$-arc in a projective plane of order $q=d d^{\prime}$ was proved by the second author in [32. This condition was used in [10] to show that five of the known projective planes of order 16 contain maximal $(52,4)$-arcs whose associated Steiner 2- $(52,4,1)$ designs are embeddable in two nonisomorphic planes of order 16 .

Construction 2.1 (Thas [28], [31], Wallis [34]). Let $\mathcal{A}$ be a maximal (qd$q+d, d)$-arc in a projective plane $\mathcal{P}$ of order $q=d d^{\prime}, 2 \leq d<q$, and let $X$ be the set of points of $\mathcal{P}$. Let $P=X \backslash \mathcal{A}$, and let $L$ be the set of all lines of $\mathcal{P}$ that intersect $\mathcal{A}$ in d points. Then $G=(P, L)$ is a partial geometry $p g(s, t, \alpha)$ with parameters (2). Similarly, the dual arc $\mathcal{A}^{\prime}$ determines a partial geometry $G^{\prime}$ with parameters $t, s, \alpha$, being the dual geometry of $G$.

We note that if $q=d d^{\prime}$, then the parameters (2) of a partial geometry associated with a maximal arc $\mathcal{A}$ of degree $d$ via Construction 2.1 can be rewritten as in eq. (44), while the numbers of points and lines (11) can be 
written as in eq. (5).

$$
\begin{gathered}
s=d\left(d^{\prime}-1\right), t=d^{\prime}(d-1), \alpha=(d-1)\left(d^{\prime}-1\right) . \\
v=(s+1)\left(d d^{\prime}+1\right), b=(t+1)\left(d d^{\prime}+1\right) .
\end{gathered}
$$

Let $d \geq 2, d^{\prime} \geq 2$ be integer numbers, and let $G=(P, L)$ be a partial geometry with parameters $s, t, \alpha$ given by eq. (44). Thus, by eq. (5), the line size $s+1$ of $G$ divides the number of points $v$, while $t+1$, that is, the line size the dual geometry $G^{\prime}$, divides $b$. A parallel class of $G$ is a set of $v /(s+1)=d d^{\prime}+1$ pairwise disjoint lines of $G$. Similarly, a parallel class of the dual geometry $G^{\prime}$ is a set of $b /(t+1)=d d^{\prime}+1$ pairwise disjoint lines of $G^{\prime}$.

Definition 2.2 Two parallel classes of $G$ (resp. $\left.G^{\prime}\right)$ are called orthogonal if they share exactly one line.

The following theorem gives an upper bound on the maximum number of pairwise orthogonal parallel classes.

Theorem 2.3 Let $G=(P, L)$ be a partial geometry $\mathrm{pg}(s, t, \alpha)$ with parameters (4), where $d \geq 2, d^{\prime} \geq 2$ are given integer numbers, and let $G^{\prime}$ be its dual partial geometry.

(a) If $C$ is a set of $m$ pairwise orthogonal parallel classes of $G$ then

$$
m \leq d\left(d d^{\prime}-d^{\prime}+1\right)
$$

and the equality $m=d\left(d d^{\prime}-d^{\prime}+1\right)$ holds if and only if every line of $G$ appears in exactly d parallel classes from $C$.

(b) If $C^{\prime}$ is a set of $m^{\prime}$ pairwise orthogonal parallel classes of $G^{\prime}$ then

$$
m^{\prime} \leq d^{\prime}\left(d^{\prime} d-d+1\right)
$$

and the equality $m^{\prime}=d^{\prime}\left(d^{\prime} d-d+1\right)$ holds if and only if every line of $G^{\prime}$ appears in exactly $d^{\prime}$ parallel classes from $C^{\prime}$.

Proof. (a) Let $k_{i}$ denote the number of parallel classes from $C$ that contain the $i$ th line $L_{i}$ of $G, 1 \leq i \leq b$, where the number $b$ of lines of $G$ is equal to $b=\left(d^{\prime}(d-1)+1\right)\left(d d^{\prime}+1\right)$ by eq. (15) and (44). Let $C_{1}, \ldots, C_{m}$ be the parallel classes from $C$. Counting in two ways the ordered pairs $\left(C_{i}, L_{t}\right)$, where $L_{t}$ belongs to $C_{i}$ gives

$$
\sum_{i=1}^{b} k_{i}=m\left(d d^{\prime}+1\right)
$$


Similarly, counting in two ways the ordered pairs $\left(\left\{C_{i}, C_{j}\right\}, L_{t}\right)$, where $L_{t}$ belongs to $C_{i}$ and $C_{j}(i \neq j)$, gives

$$
\sum_{i=1}^{b} k_{i}\left(k_{i}-1\right)=m(m-1)
$$

Adding equations (8) and (9) gives

$$
\sum_{i=1}^{b} k_{i}^{2}=m\left(m+d d^{\prime}\right)
$$

Applying the Cauchy-Schwarz inequality, we have

$$
\left(\sum_{i=1}^{b} k_{i}\right)^{2}=m^{2}\left(d d^{\prime}+1\right)^{2} \leq b \sum_{i=1}^{b} k_{i}^{2}=\left(d^{\prime}(d-1)+1\right)\left(d d^{\prime}+1\right) m\left(m+d d^{\prime}\right),
$$

whence

$$
m\left(d d^{\prime}+1\right) \leq\left(d^{\prime}(d-1)+1\right)\left(m+d d^{\prime}\right)
$$

Solving the last inequality for $m$ implies (6) $)$. Clearly, the equality

$$
m=d\left(d d^{\prime}-d^{\prime}+1\right)
$$

holds if and only if

$$
k_{1}=k_{2}=\cdots=k_{b}=\frac{1}{b} \sum_{i=1}^{b} k_{i}=\frac{d\left(d d^{\prime}-d^{\prime}+1\right)\left(d d^{\prime}+1\right)}{\left(d^{\prime} d-d^{\prime}+1\right)\left(d d^{\prime}+1\right)}=d .
$$

This completes the proof of part (a).

(b) Switch $d$ and $d^{\prime}, b$ and $v, s$ and $t$ in the proof of part (a).

The following theorem shows that a partial geometry $G$ with parameters (4) arises from a maximal arc if and only if $G$ and its dual geometry $G^{\prime}$ both meet the bounds of Theorem 2.3.

Theorem 2.4 Let $d \geq 2, d^{\prime} \geq 2$ be integer numbers, and let $G$ be a partial geometry $\mathrm{pg}(s, t, \alpha)$ with parameters (4).

A necessary and sufficient condition for $G$ to be associated with a maximal arc of degree $d$ in a projective plane $\mathcal{P}$ of order $q=d d^{\prime}$ is that the following two conditions hold:

(i) $G$ admits a set of pairwise orthogonal parallel classes that meets the upper bound (6) of Theorem [2.3, part (a).

(ii) The dual geometry $G^{\prime}$ admits a set of pairwise orthogonal parallel classes that meets the upper bound (7) of Theorem [2.3, part (b). 
Proof. Assume that $\mathcal{P}$ is a projective plane of order $q=d d^{\prime}$ with a maximal $(q d-q+d, d)$-arc $\mathcal{A}$, and let $\mathcal{A}^{\prime}$ be the dual $\left(q d^{\prime}-q+d^{\prime}, d^{\prime}\right)$-arc. Let $G$ be the partial geometry with parameters given by (44) and (5) that arises from $\mathcal{A}$ via Construction 2.1. Thus, if $X$ is the point set of $\mathcal{P}$ then $X \backslash \mathcal{A}$ is the set of points of $G$.

A pencil is a set of $q+1$ lines of $\mathcal{P}$ that pass through a point $x$. If $x$ is a point from the arc $\mathcal{A}$, the pencil through $x$ determines

$$
v /(s+1)=q+1=d d^{\prime}+1
$$

pairwise disjoint lines of $G$ via Construction [2.1, and these $q+1$ lines of $G$ form a parallel class. Since every two points $x, y \in \mathcal{A}, x \neq y$ are incident with exactly one line $l$ of $\mathcal{P}$, the pencils through $x$ and $y$ determine two parallel classes $C_{1}, C_{2}$ of lines of $G$ that share exactly one line, being the restriction of $l$ on the point set $X \backslash \mathcal{A}$ of $G$. Thus $C_{1}$ and $C_{2}$ are orthogonal. It follows that the $q d-q+d=d\left(d d^{\prime}-d^{\prime}+1\right)$ points of $\mathcal{A}$ determine a set of $d\left(d d^{\prime}-d^{\prime}+1\right)$ pairwise orthogonal classes of lines of $G$ that meets the bound (6) of Theorem 2.3 .

Similarly, the $d^{\prime}\left(d^{\prime} d-d+1\right)$ points of $\mathcal{A}^{\prime}$ determine a set of $d^{\prime}\left(d^{\prime} d-d+1\right)$ pairwise orthogonal parallel classes of lines of the dual geometry $G^{\prime}$ that meets the bound (7) of Theorem 2.3 .

Suppose now that $G=(P, L)$ is a partial geometry with parameters (4) for some integers $d \geq 2, d^{\prime} \geq 2$, and assume that $G$ and its dual geometry $G^{\prime}$ satisfy the conditions (i) and (ii) respectively. Then one can construct a projective plane $\mathcal{P}$ of order $q=d d^{\prime}$ as follows.

Let $C=\left\{C_{1}, \ldots, C_{m}\right\}$ be a set of $m$ pairwise orthogonal parallel classes of lines of $G$, where $m=d\left(d d^{\prime}-d^{\prime}+1\right)$ meets the bound ([6), and let $C^{\prime}=\left\{C_{1}^{\prime}, \ldots, C_{m^{\prime}}^{\prime}\right\}$ be a set of $m^{\prime}$ pairwise orthogonal parallel classes of lines of the dual geometry $G^{\prime}$, where $m^{\prime}=d^{\prime}\left(d^{\prime} d-d+1\right)$ meets the bound (7). According to Theorem 2.3 , every line of $G$ appears in exactly $d$ parallel classes from $C$, and every line of $G^{\prime}$ appears in exactly $d^{\prime}$ parallel classes from $C^{\prime}$. By (44) and (5), every parallel class $C_{i} \in C$, as well as every parallel class $C_{i}^{\prime} \in C^{\prime}$, consists of $q+1$ pairwise disjoint lines.

We define an incidence structure $\mathcal{P}$ with a set of points $X$ and a collection of lines $\mathcal{L}$, where $X$ consists of the $v=|P|$ points of $G$ plus $m=d\left(d d^{\prime}-d^{\prime}+1\right)$ new points labeled by the $m$ parallel classes from $C$, and $\mathcal{L}$ consists of $b=|L|$ lines labeled by the lines of $G$ plus $m^{\prime}=d^{\prime}\left(d^{\prime} d-d+1\right)$ lines labeled by the $m^{\prime}$ parallel classes from $C^{\prime}$. Thus, by (4) and (5), $\mathcal{P}$ has $q^{2}+q+1$ points and $q^{2}+q+1$ lines.

A line $l^{*}$ of $\mathcal{P}$ which is labeled by a line $l$ of $G$ consists of the $s+1$ points of $l$ and $d$ points labeled by the $d$ parallel classes from $C$ that contain the line $l$. It follows from (4) that $l^{*}$ is incident with $s+1+d=q+1$ points. 
A line $l_{i}^{\prime}$ of $\mathcal{P}$ which is labeled by a parallel class $C_{i}^{\prime} \in C^{\prime}$, consists of the $q+1$ points of $G$ that correspond to the $q+1$ lines of $G^{\prime}$ belonging to $C_{i}^{\prime}$, $1 \leq i \leq m^{\prime}$. It follows from (4) that every point of $\mathcal{P}$ which is also a point of $G$ is incident with $t+1+d^{\prime}=q+1$ lines of $\mathcal{P}$, and every point of $\mathcal{P}$ which is labeled by a parallel class $C_{i}$ of $G$ is also incident with $q+1$ lines of $\mathcal{P}$.

Thus, $\mathcal{P}=(X, \mathcal{L})$ is an incidence structure with $|X|=q^{2}+q+1$ points and $|\mathcal{L}|=q^{2}+q+1$ lines, such that every line is incident with $q+1$ points, and every point is incident with $q+1$ lines. To show that $\mathcal{P}$ is a projective plane of order $q$, it is sufficient to check that every two lines of $\mathcal{L}$ meet in exactly one point of $X$.

Any two distinct parallel classes $C_{i}^{\prime}, C_{j}^{\prime} \in C^{\prime}$ share exactly one line $l^{\prime}$ of $G^{\prime}$ due to the orthogonality condition. Consequently, the two lines of $\mathcal{P}$ labeled by $C_{i}^{\prime}$ and $C_{j}^{\prime}$ meet in exactly one point $x \in P$, being the point of $G$ that corresponds to the line $l^{\prime}$ of $G^{\prime}$.

A line $l^{*}$ of $\mathcal{P}$ which is labeled by a line $l$ of $G$ meets any line of $\mathcal{P}$ which is labeled by a parallel class $C_{i}^{\prime} \in C^{\prime}$ in exactly one point, because $l$ is incident with exactly one of the $q+1$ points of $G$ that correspond to the $q+1$ parallel lines of $G^{\prime}$ belonging to $C_{i}^{\prime}$.

Let $l_{1}^{*}, l_{2}^{*}$ be lines of $\mathcal{P}$ that are labeled by two distinct lines $l_{1}, l_{2}$ of $G$.

If $l_{1}$ and $l_{2}$ belong to a parallel class $C_{i} \in C$, then $l_{1}^{*}$ and $l_{2}^{*}$ meet in exactly one point, being the point of $\mathcal{P}$ labeled by $C_{i}$. On the other hand, if $l_{1}$ and $l_{2}$ are two distinct lines of $G$ that belong to two different parallel classes from $C$, then $l_{1}^{*}$ and $l_{2}^{*}$ cannot share any point labeled by a parallel class from $C$, and can possibly share at most one point, being a point of $G$.

To prove that every two lines of $\mathcal{P}$ share a point, we count the set of ordered pairs

$$
S=\left\{\left(x,\left\{l_{i}^{*}, l_{j}^{*}\right\}\right)\right\},
$$

where $x \in X$ is a point of $\mathcal{P}$, and $l_{i}^{*}, l_{j}^{*} \in \mathcal{L}, 1 \leq i<j \leq q^{2}+q+1$, are two distinct lines of $\mathcal{P}$ that are both incident with $x$.

Since for every two distinct lines $l_{i}^{*}, l_{j}^{*}$ there is at most one point $x$ incident with both $l_{i}^{*}$ and $l_{j}^{*}$, we have

$$
|S| \leq\left(\begin{array}{c}
q^{2}+q+1 \\
2
\end{array}\right)
$$

On the other hand, since every point $x$ belongs to $q+1$ lines, there are $\left(\begin{array}{c}q+1 \\ 2\end{array}\right)$ pairs of lines that are incident with $x$. Thus, counting the ordered pairs from $S$ by ranging $x$ over the set of all $q^{2}+q+1$ points implies that $|S|$ is given by eq. (11).

$$
|S|=\left(q^{2}+q+1\right)\left(\begin{array}{c}
q+1 \\
2
\end{array}\right)=\frac{\left(q^{2}+q+1\right)(q+1) q}{2} .
$$


Since

$$
\frac{\left(q^{2}+q+1\right)(q+1) q}{2}=\left(\begin{array}{c}
q^{2}+q+1 \\
2
\end{array}\right),
$$

it follows from (10), (11) and (12) that each pair of distinct lines share exactly one point, thus $\mathcal{P}$ is a projective plane of order $q=d d^{\prime}$.

Since every line of $\mathcal{P}$ which is labeled by a line of $G$ meets the set $\mathcal{A}$ of $m=d\left(d d^{\prime}-d^{\prime}+1\right)$ points labeled by the parallel classes from $C$ in exactly $d$ points, and every line of $\mathcal{P}$ which is labeled by a parallel class from $C^{\prime}$ is disjoint from $\mathcal{A}$, the set $\mathcal{A}$ is a maximal $\left(d\left(d d^{\prime}-d^{\prime}+1\right), d\right)$-arc in $\mathcal{P}$. Similarly, the $m^{\prime}=d^{\prime}\left(d^{\prime} d-d+1\right)$ lines of $\mathcal{P}$ labeled by the parallel classes of $C^{\prime}$, determine a maximal $\left(d^{\prime}\left(d^{\prime} d-d+1\right), d^{\prime}\right)$-arc $\mathcal{A}^{\prime}$ in the dual plane of $\mathcal{P}$, and $\mathcal{A}^{\prime}$ is the dual arc of $\mathcal{A}$. $\square$.

Note 1 In all known partial geometries with parameters of the form (4), $d$ and $d^{\prime}$ are both powers of 2 . It is an interesting open question whether partial geometries for other values of $d$ and $d^{\prime}$ may exist.

For example, it is not known if a partial geometry $p g(6,6,2),\left(d=d^{\prime}=3\right)$ exists or not. However, a partial geometry with these parameters cannot be associated with a maximal $(21,3)$-arc in a projective plane of order 9 , because no such maximal arcs exist in any of the four projective planes of order 9 [20].

Another open small parameter set is $s=12, t=10, \alpha=8$, that corresponds to $d=3$ and $d^{\prime}=5$. The existence of a partial geometry $p g(12,10,8)$ that satisfies the conditions of Theorem 2.4 would imply the existence of a projective plane of order 15 with maximal arcs of degree 3 and 5 .

\section{$3 \quad$ Partial geometries $p g(4,6,3)$}

A strongly regular graph $\Gamma$ with parameters $n, k, \lambda, \mu(\operatorname{or} \operatorname{srg}(n, k, \lambda, \mu))$ is an undirected graph without loops or multiple edges, having the following properties:

- $\Gamma$ has $n$ vertices.

- Every vertex has exactly $k$ neighbors.

- Every two adjacent vertices have exactly $\lambda$ common neighbors.

- Every two nonadjacent vertices have exactly $\mu$ common neighbors.

The complementary graph $\bar{\Gamma}$ of a strongly regular graph $\Gamma$ with parameters $n, k, \lambda, \mu$ is also strongly regular, with parameters $\bar{n}, \bar{k}, \bar{\lambda}, \bar{\mu}$ given by (13).

$$
\bar{n}=n, \bar{k}=n-1-k, \bar{\lambda}=n-2 k+\mu-2, \bar{\mu}=n-2 k+\lambda .
$$


Strongly regular graphs were introduced by Bose in the same paper [4], where he introduced partial geometries.

The eigenvalues of the $(0,1)$-adjacency matrix of a strongly regular graph $\Gamma$ with parameters $n, k, \lambda, \mu$, as well as their multiplicities, are easily expressed in terms of the graph parameters [4]: $k$ is a simple eigenvalue, and up to multiplicity, there are two more eigenvalues $\rho_{1}, \rho_{2}$, being the solutions of the quadratic equation (14).

$$
x^{2}+(\mu-\lambda) x+\mu-k=0 .
$$

The following statement is a special case of a more general result due to Hoffman [18] that applies to regular graphs.

Theorem 3.1 (Hoffman bound) Let $\Gamma$ be a strongly regular graph with parameters $n, k, \lambda, \mu$, and let $\rho$ be the smallest eigenvalue of the (0,1)-adjacency matrix of $\Gamma$, being the negative root of equation (14). The size of any coclique $C$ of $\Gamma$ satisfies the inequality

$$
c=|C| \leq \frac{n(-\rho)}{k-\rho},
$$

and the equality holds if and only if every vertex outside $C$ is adjacent to exactly

$$
d=\frac{k c}{n-c}
$$

vertices of $C$.

If $G=(P, L)$ is a partial geometry $p g(s, t, \alpha)$ with point set $P$ and line set $L$, the point graph $\Gamma_{P}$ of $G$ is the graph with vertex set $P$, where two vertices are adjacent if the corresponding points of $G$ are collinear. The line graph $\Gamma_{L}$ of $G$ is the graph having as vertices the lines of $G$, where two lines are adjacent if they share a point. Both $\Gamma_{P}$ and $\Gamma_{L}$ are strongly regular graphs [4]. The parameters $n, k, \lambda, \mu$ of $\Gamma_{P}$ are expressed in terms of $s, t, \alpha$ as in eq. (16), while the parameters $n^{\prime}, k^{\prime}, \lambda^{\prime}, \mu^{\prime}$ of $\Gamma_{L}$ are given by eq. (17).

$$
\begin{aligned}
& n=(s+1)(s t+\alpha) / \alpha, k=s(t+1), \lambda=s-1+t(\alpha-1), \mu=\alpha(t+1) . \\
& n^{\prime}=(t+1)(s t+\alpha) / \alpha, k^{\prime}=t(s+1), \lambda^{\prime}=t-1+s(\alpha-1), \mu^{\prime}=\alpha(s+1) .
\end{aligned}
$$

A strongly regular graph $\Gamma$ whose parameters $n, k, \lambda, \mu$ can be written as in eq. (16) for some integers $s, t, \alpha$ is called pseudo-geometric, and $\Gamma$ is called geometric if there exists a partial geometry $G$ with parameters $s, t, \alpha$ such that $\Gamma$ is the point graph of $G$; otherwise $\Gamma$ is non-geometric.

Applying the Hoffman bound (15) from Theorem 3.1 to the complementary graph $\bar{\Gamma}$ of a pseudo-geometric graph $\Gamma$ with parameters (16) implies that every clique of $\Gamma$ is of size smaller than or equal to $s+1$, and every clique $C$ of maximum size $s+1$ has the property that every vertex outside $C$ is adjacent to exactly $\alpha$ vertices from $C$. 
Theorem 3.2 (Bose [4]). A pseudo-geometric strongly regular graph $\Gamma$ with parameters (16) is geometric if and only if $\Gamma$ possesses a set of $b=(t+1)($ st + $\alpha) / \alpha$ cliques of size $s+1$, every two of which share at most one vertex.

Next, we consider partial geometries with parameters $s, t, \alpha$ of the form (4) for some integer $d \geq 2$ and $d^{\prime}=2$, namely

$$
s=d, t=2 d-2, \alpha=d-1 .
$$

The parameters (16) of the point graph of a partial geometry with parameters (18) are given by eq. (19).

$$
n=(d+1)(2 d+1), k=d(2 d-1), \lambda=(d-1)(2 d-3), \mu=(d-1)(2 d-1) .
$$

The triangular graph $T(m)$, where $m \geq 4$ is an integer, has as vertices the unordered 2-subsets of $\{1,2, \ldots, m\}$, where two distinct 2 -subsets are adjacent in $T(m)$ whenever they are not disjoint. The graph $T(m)$ is strongly regular with parameters (20), while its complementary graph $\bar{T}(m)$ has parameters (21).

$$
\begin{gathered}
n=\left(\begin{array}{c}
m \\
2
\end{array}\right), k=2(m-2), \lambda=m-2, d=4 . \\
\bar{n}=\left(\begin{array}{c}
m \\
2
\end{array}\right), \bar{k}=\left(\begin{array}{c}
m-2 \\
2
\end{array}\right), \bar{\lambda}=\left(\begin{array}{c}
m-4 \\
2
\end{array}\right), \bar{\mu}=\left(\begin{array}{c}
m-3 \\
2
\end{array}\right) .
\end{gathered}
$$

Let $d \geq 2$ be an integer. By (21), the parameters of $\bar{T}(2 d+2)$ are

$$
\bar{n}=(d+1)(2 d+1), \bar{k}=d(2 d-1), \bar{\lambda}=(d-1)(2 d-3), \bar{\mu}=(d-1)(2 d-1) .
$$

Since the parameters (22) and (19) coincide, the graph $\bar{T}(2 d+2)$ is pseudogeometric for $s, t, \alpha$ given by (18). It is known that every strongly regular graph with parameters (20) is isomorphic to $T(m)$, except when $m=8$, in which case in addition to $T(8)$, there are three other graphs [6], [17], known as the Chang graphs.

The question about the values of $d$ for which a strongly regular graph with parameters (22) is geometric has been settled in the following cases:

1. $d=2^{i}, i \geq 0$. The graph $\bar{T}\left(2^{i+1}+2\right)$ is geometric, and the corresponding partial geometry arises from a hyperoval in a projective plane of order $2^{i+1}$ via Construction 2.1 .

2. $d=3$. Neither $\bar{T}(8)$, nor any of the Chang graphs is geometric, thus, a partial geometry with parameters $s=3, t=4, \alpha=2$ does not exist (De Clerck [7]). 
3. $d=4$. The graph $\bar{T}(10)$ is geometric, and up to isomorphism, there exist exactly two partial geometries $p g(4,6,3)$ (Mathon [21]). The dual geometry of one of these two geometries arises from a hyperoval in the projective plane of order $8, P G(2,8)$, via Construction 2.1, while the dual of the second geometry is not associated with any hyperoval in $P G(2,8)$.

4. $d=5$. The graph $\bar{T}(12)$ is not geometric, thus a partial geometry $p g(5,8,4)$ does not exist (Lam, Thiel, Swiercz, and McKay [19]).

We will give a brief description of the two partial geometries $p g(4,6,3)$ having $\bar{T}(10)$ as point graph. A convenient way to describe a partial geometry $G_{1}$ associated with a maximal arc of degree 4 (dual arc of a hyperoval) in $P G(2,8)$ is by using a collineation $f$ of order 9 acting fixed-point-free on the set of points $P=\{1,2, \ldots, 45\}$, as

$$
f_{P}=(1,2, \ldots, 9)(10, \ldots, 18) \cdots(37, \ldots, 45),
$$

and on the set of 63 lines $L=\left\{L_{1}, \ldots, L_{63}\right\}$, as

$$
f_{L}=\left(L_{1}, \ldots, L_{9}\right)\left(L_{10}, \ldots, L_{18}\right) \cdots\left(L_{55}, \ldots, L_{63}\right) .
$$

Representatives of the seven orbits of lines are listed in Table 1. The order of the full automorphism group of $G_{1}$ is 1512 , which is also the order of the stabilizer of a hyperoval in $P G(2,8)$. The 2 -rank (that is, the rank over the finite field of order 2 ) of the incidence matrix of $G_{1}$ is equal to 28 . We note that 28 is also the 2-rank of the incidence matrix of the projective plane of order 8 .

We define a graph $\mathcal{L}$ having as vertices the lines of $G_{1}$, where two lines are adjacent in $\mathcal{L}$ if they are disjoint, or in other words, $\mathcal{L}$ is the complementary graph of the line graph of $G_{1}$. Clearly, the maximum clique size in $\mathcal{L}$ is $45 / 5=9$, and every clique of size 9 is a parallel class of lines. Using the clique finding algorithm Cliquer developed by Niskanen and Östergård [24, one quickly finds by computer that $\mathcal{L}$ contains exactly 28 cliques of size 9 , every two sharing one vertex, that give a set $C$ of 28 pairwise orthogonal parallel classes of lines, listed in Table 2, where the indices of the lines in each parallel class are given. Note that 28 is equal to the upper bound (6) of Theorem 2.3, (a). Similarly, the parallel classes of lines of the dual geometry $G_{1}^{\prime}$, each consisting of nine pairwise disjoint lines of $G_{1}^{\prime}$, can be found as cliques of size 9 in the complementary graph of the line graph of $G_{1}^{\prime}$, or equivalently, the complementary graph of the point graph of $G_{1}$, the latter being isomorphic to the triangular graph $T(10)$. Clearly, there are exactly ten such cliques meeting pairwise in one vertex. The set $C^{\prime}$ of ten specific cliques found by Cliquer are listed in Table [3, where each line of the dual 
geometry $G_{1}^{\prime}$ is labeled by a point of $G_{1}$. We note that the set of ten pairwise orthogonal parallel classes meets the upper bound (7) of Theorem 2.3 (b). Thus, a projective plane of order 8 is uniquely determined from $G_{1}$ and the sets of parallel classes $C$ and $C^{\prime}$ by the construction described in the proof of Theorem 2.4.

The second partial geometry $G_{2}$ with parameters $s=4, t=6, \alpha=3$, found by Mathon [21], can be described in terms of a permutation $g$ of order 6 and representatives of the line orbits under the group generated by $g$ as follows. The permutation $g$ acts on the 45 points as

$$
g_{P}=(1, \ldots, 6)(7, \ldots, 12) \cdots(31, \ldots, 36)(37,38,39)(40,41)(42,43)(44)(45)
$$

and $g$ acts on the set of 63 lines $\left\{l_{1}, \ldots, l_{63}\right\}$ as

$$
g_{L}=\left(l_{1}, \ldots, l_{6}\right) \cdots\left(l_{49}, \ldots, l_{54}\right)\left(l_{55}, l_{56}, l_{57}\right)\left(l_{58}, l_{59}, l_{60}\right)\left(l_{61}, l_{62}\right)\left(l_{63}\right) .
$$

We take as line orbit representative the first line from each orbit. These representatives are given in Table 4. The order of the full automorphism group of $G_{2}$ is 216 [21]. The 2-rank of the incidence matrix of $G_{2}$ is equal to 34 , thus, $G_{2}$ does not arise from a maximal arc in a projective plane of order 8 , because the 2-rank of the incidence matrix of the (unique up to isomorphism) projective plane of order 8 is equal to 28 . To find all parallel classes of lines of $G_{2}$, we consider each parallel class as a clique of size 9 in the complementary graph of the line graph of $G_{2}$. Using Cliquer, we found that $G_{2}$ contains only one parallel class of lines. Thus, $G_{2}$ does not satisfy the first codition of Theorem 2.4, and consequently, this partial geometry is not associated with a maximal arc of degree 4 (dual arc of a hyperoval) in a projective plane of order 8 . The indices of the lines form the unique parallel class are

$$
25,26,27,28,29,30,61,62,63
$$

and the points of these lines are listed in Table 5. The parallel classes of lines of the dual geometry $G_{2}^{\prime}$, each consisting of nine pairwise disjoint lines of $G_{2}^{\prime}$, can be found as maximal cliques of size 9 in the complementary graph of the point graph of $G_{2}$, being isomorphic to the triangular graph $T(10)$. There are exactly ten such cliques, and the cliques found by Cliquer are listed in Table 6, where each line of the dual geometry $G_{2}^{\prime}$ is labeled by a point of $G_{2}$. The partial geometries $p g(4,6,3)$ having $\bar{T}(10)$ as a point graph were classified by Mathon [21] by enumerating and classifying up to isomorphism the collections of 635 -cliques of $\bar{T}(10)$ meeting pairwise in at most one vertex. A pencil through a point $x$ of a partial geometry $p g(4,6,3)$ is the set of lines though $x$. To reduce the search, Mathon [21] enumerated and classified the pencils through a pair of vertices of $\bar{T}(10)$, and analyzed their completion to a partial geometry. An alternative construction of Mathon's partial geometry 


\begin{tabular}{|l|lllll|}
\hline$L_{1}$ & 9 & 11 & 22 & 34 & 42 \\
\hline$L_{10}$ & 1 & 2 & 18 & 22 & 30 \\
\hline$L_{19}$ & 1 & 3 & 15 & 27 & 42 \\
\hline$L_{28}$ & 11 & 12 & 14 & 19 & 30 \\
\hline$L_{37}$ & 10 & 19 & 40 & 42 & 45 \\
\hline$L_{46}$ & 1 & 5 & 19 & 32 & 41 \\
\hline$L_{55}$ & 19 & 28 & 29 & 34 & 44 \\
\hline
\end{tabular}

Table 1: Line orbit representatives of $G_{1}$

based on over-large Steiner systems was proposed recently by Reichard and Woldar [27].

Using Cliquer, we enumerated and classified up to isomorphism all partial geometries $p g(4,6,3)$ by computing all maximal cliques in a graph $\Omega$ having as vertices the $10 ! /\left(2^{5} 5 !\right)=9455$-cliques of $\bar{T}(10)$, where two 5 -cliques of $\bar{T}(10)$ are adjacent in $\Omega$ if and only if they share at most one vertex. Clearly, any set of 635 -cliques of $\bar{T}(10)$ that is a clique in $\Omega$ is the line set of a partial geometry $p g(4,6,3)$, and vice versa. It took Cliquer less than one minute on a personal computer MacBook Pro to find all cliques of size 63 in $\Omega$, an their number is 19200 .

According to the order of the stabilizer of a 63 -clique in the symmetric group $S_{10}$, which acts as the full automorphism group of both $\bar{T}(10)$ and $\Omega$, the 19200 63-cliques of $\Omega$ are partitioned into two sets: 2400 cliques with a stabilizer of order 1512, and the remaining 16800 63-cliques with a stabilizer of order 216. Since

$$
\frac{10 !}{1512}+\frac{10 !}{216}=2400+16800=19200,
$$

it follows that the 19200 distinct partial geometries $p g(4,6,3)$ having $\bar{T}(10)$ as point graph, are split into two ismoprphism classes, one geometry with automorphism group of order 1512, being isomorphic to the geometry arising from a maximal arc of degree 4 in $P G(2,8)$, and a second geometry with automorphism group of order 216, which is isomorphic to Mathon's geometry. Thus, our computations give an alternative and independent confirmation of Mathon's classification [21], and Theorem 2.4 provides an alternative explanation why the dual of Mathon's geometry does not arise from a hyperoval in a projective plane of order 8 . 


\begin{tabular}{|lllllllll|}
\hline 7 & 10 & 13 & 17 & 36 & 41 & 42 & 48 & 62 \\
2 & 12 & 14 & 17 & 31 & 37 & 45 & 52 & 57 \\
3 & 13 & 15 & 18 & 32 & 37 & 38 & 53 & 58 \\
5 & 26 & 28 & 33 & 45 & 47 & 48 & 50 & 58 \\
2 & 23 & 30 & 34 & 42 & 47 & 53 & 54 & 55 \\
6 & 12 & 16 & 18 & 35 & 40 & 41 & 47 & 61 \\
3 & 17 & 19 & 22 & 23 & 28 & 44 & 59 & 61 \\
2 & 16 & 21 & 22 & 27 & 36 & 43 & 58 & 60 \\
6 & 11 & 22 & 25 & 26 & 31 & 38 & 55 & 62 \\
9 & 14 & 19 & 20 & 25 & 34 & 41 & 56 & 58 \\
9 & 10 & 12 & 15 & 29 & 43 & 44 & 50 & 55 \\
9 & 21 & 28 & 32 & 40 & 51 & 52 & 54 & 62 \\
4 & 25 & 32 & 36 & 44 & 46 & 47 & 49 & 57 \\
6 & 27 & 29 & 34 & 37 & 48 & 49 & 51 & 59 \\
7 & 19 & 30 & 35 & 38 & 49 & 50 & 52 & 60 \\
8 & 11 & 14 & 18 & 28 & 42 & 43 & 49 & 63 \\
7 & 12 & 23 & 26 & 27 & 32 & 39 & 56 & 63 \\
5 & 11 & 15 & 17 & 34 & 39 & 40 & 46 & 60 \\
8 & 20 & 31 & 36 & 39 & 50 & 51 & 53 & 61 \\
4 & 10 & 14 & 16 & 33 & 38 & 39 & 54 & 59 \\
5 & 10 & 21 & 24 & 25 & 30 & 37 & 61 & 63 \\
4 & 18 & 20 & 23 & 24 & 29 & 45 & 60 & 62 \\
3 & 24 & 31 & 35 & 43 & 46 & 48 & 54 & 56 \\
8 & 13 & 19 & 24 & 27 & 33 & 40 & 55 & 57 \\
1 & 2 & 3 & 4 & 5 & 6 & 7 & 8 & 9 \\
1 & 22 & 29 & 33 & 41 & 46 & 52 & 53 & 63 \\
1 & 15 & 20 & 21 & 26 & 35 & 42 & 57 & 59 \\
1 & 11 & 13 & 16 & 30 & 44 & 45 & 51 & 56 \\
\hline
\end{tabular}

Table 2: Parallel classes of $G_{1}$

\begin{tabular}{|llllllllll|}
\hline 19 & 20 & 21 & 22 & 23 & 24 & 25 & 26 & 27 \\
6 & 9 & 10 & 15 & 25 & 30 & 32 & 43 & 44 \\
4 & 7 & 13 & 17 & 23 & 28 & 30 & 41 & 42 \\
2 & 8 & 12 & 17 & 27 & 32 & 34 & 37 & 45 \\
2 & 5 & 11 & 15 & 21 & 28 & 35 & 39 & 40 \\
5 & 8 & 14 & 18 & 24 & 29 & 31 & 42 & 43 \\
3 & 9 & 13 & 18 & 19 & 33 & 35 & 37 & 38 \\
3 & 6 & 12 & 16 & 22 & 29 & 36 & 40 & 41 \\
1 & 4 & 10 & 14 & 20 & 34 & 36 & 38 & 39 \\
1 & 7 & 11 & 16 & 26 & 31 & 33 & 44 & 45 \\
\hline
\end{tabular}

Table 3: Parallel classes of $G_{1}^{\prime}$ 


\begin{tabular}{|l|llllll|}
\hline$l_{1}$ & 14 & 22 & 30 & 35 & 40 \\
$l_{7}$ & 12 & 25 & 34 & 38 & 40 \\
$l_{13}$ & 1 & 21 & 28 & 35 & 45 \\
$l_{19}$ & 1 & 13 & 26 & 27 & 43 \\
$l_{25}$ & 1 & 9 & 18 & 30 & 34 \\
$l_{31}$ & 1 & 8 & 23 & 39 & 42 \\
$l_{37}$ & 1 & 11 & 12 & 22 & 32 \\
$l_{43}$ & 1 & 16 & 17 & 36 & 38 \\
$l_{49}$ & 1 & 10 & 15 & 24 & 33 \\
$l_{55}$ & 7 & 10 & 27 & 30 & 44 \\
$l_{58}$ & 13 & 16 & 20 & 23 & 44 \\
$l_{61}$ & 19 & 21 & 23 & 40 & 43 \\
$l_{63}$ & 37 & 38 & 39 & 44 & 45 \\
\hline
\end{tabular}

Table 4: Line orbit representatives of $G_{2}$

\begin{tabular}{|llllll|}
\hline 1 & 9 & 18 & 30 & 34 \\
2 & 10 & 13 & 25 & 35 \\
3 & 11 & 14 & 26 & 36 \\
4 & 12 & 15 & 27 & 31 \\
5 & 7 & 16 & 28 & 32 \\
6 & 8 & 17 & 29 & 33 \\
19 & 21 & 23 & 40 & 43 \\
20 & 22 & 24 & 41 & 42 \\
37 & 38 & 39 & 44 & 45 \\
\hline
\end{tabular}

Table 5: The unique parallel class of $G_{2}$

\begin{tabular}{|llllllllll|}
\hline 31 & 32 & 33 & 34 & 35 & 36 & 42 & 43 & 44 \\
2 & 8 & 15 & 20 & 21 & 26 & 30 & 32 & 38 \\
5 & 11 & 18 & 23 & 24 & 27 & 29 & 35 & 38 \\
6 & 12 & 13 & 19 & 24 & 28 & 30 & 36 & 39 \\
4 & 10 & 17 & 22 & 23 & 26 & 28 & 34 & 37 \\
8 & 10 & 12 & 14 & 16 & 18 & 41 & 43 & 45 \\
1 & 7 & 14 & 19 & 20 & 25 & 29 & 31 & 37 \\
7 & 9 & 11 & 13 & 15 & 17 & 40 & 42 & 45 \\
1 & 2 & 3 & 4 & 5 & 6 & 40 & 41 & 44 \\
3 & 9 & 16 & 21 & 22 & 25 & 27 & 33 & 39 \\
\hline
\end{tabular}

Table 6: Parallel classes of $G_{2}^{\prime}$ 


\section{Partial geometries arising from planes of small order}

The upper bounds (6) and (7) of Theorem 2.3 apply only to sets of parallel classes that are pairwise orthogonal. It is an interesting open question if the total number of parallel classes of any partial geometry with parameters (4) or its dual geometry can exceed any of these bounds.

Motivated by this question, we examined the parallel classes of lines in partial geometries arising from maximal arcs of degree $d, 2 \leq d<q$ in the projective planes of even order $q=2^{r} \leq 16$ via Construction 2.1 .

The smallest $q$ that meets the condition $2 \leq d<q$ is $q=4$. In this case $d=d^{\prime}=2$, and any maximal arc is a hyperoval. The projective plane $P G(2,4)$ of order 4 contains 168 hyperovals, all being in one orbit under the collineation group of the plane. Thus, up to isomorphism, there is a unique partial geometry $p g(2,2,1)$ associated with a hyperoval in $P G(2,4)$, known also as the generalized quadrangle $W(2)$ (Payne and Thas [25]). The total number of parallel classes of lines of $W(2)$ is exactly six, and the six parallel classes are pairwise orthogonal. We note that the 2-rank of the incidence matrix of $W(2)$ is 10 , and is equal to the 2-rank of the incidence matrix of the projective plane of order 4 .

If $q=8$, the possible degrees of maximal arcs are $d=2$ and $d=4$. All hyperovals in $P G(2,8)$ are projectively equivalent. Thus, up to isomorphism, there is a unique partial geometry $p g(6,4,3)$ arising from a hyperoval in $P G(2,8)$, being isomorphic to the dual of the partial geometry $G_{1}$ from Section 3, while every partial geometry $p g(4,6,3)$ arising from a maximal arc of degree 4 in $P G(2,8)$ is isomorphic to $G_{1}$. It was shown in Section 3 that $G_{1}$ has a total of exactly 28 parallel classes of lines, every two being orthogonal, and its dual geometry has exactly 10 parallel classes of lines, every two being orthogonal. As we mentioned in Section 3, the 2-rank of the incidence matrix of $G_{1}$ is 28 , which is also the 2-rank of the incidence matrix of the projective plane of order 8 .

If $q=16$, the possible degrees of a maximal arc are $d=2, d=4$, and $d=8$. If $d=2$, a maximal arc of degree 2 , that is, a hyperoval $H$, gives rise to a partial geometry $p g(14,8,7)$, while the dual arc of $H$ is of degree 8 and gives rise to the dual partial geometry with parameters $p g(8,14,7)$. If $d=4$, a maximal arc of degree 4 and its dual arc of degree 4 give rise to two not necessarily isomorphic partial geometries with parameters $p g(12,12,9)$.

It follows from Theorem 2.4 that a partial geometry $p g(14,8,7)$ associated with a hyperoval in a projective plane of order 16 must have a set of 18 pairwise orthogonal parallel classes of lines, and its dual geometry must have 120 pairwise orthogonal parallel classes of lines, while any $p g(12,12,9)$ arising

from a maximal arc of degree 4 , as well as its dual geometry each must have 
a set of 52 pairwise orthogonal parallel classes.

Up to duality, there are 22 nonisomorphic projective planes of order 16 that are known currently: four planes are self-dual and nine planes are not self-dual (see [23], 26]). All hyperovals in the known 22 projective planes of order 16 were enumerated and classified up to equivalence 2 by Penttila, Royle, and Simpson [26]: altogether, there are 93 equivalence classes of hyperovals in the 22 planes, and by duality, 93 equivalence classes of maximal arcs of degree 8 . The specific line sets of the known projective planes of order 16 and representatives of the equivalence classes of hyperovals were graciously provided to the second author by Gordon F. Royle.

Using Magma [5] and Cliquer [24], we checked by computer that the 93 equivalence classes of hyperovals give rise to 93 nonisomorphic partial geometries $p g(14,8,7)$ arising from hyperovals, and, by duality, 93 nonisomorphic $p g(8,14,7)$ arising from maximal arcs of degree 8 in projective planes of order 16. We computed all parallel classes of lines, and in all cases, a partial geometry $p g(14,8,7)$ associated with a hyperoval has exactly 18 parallel classes, while its dual geometry has exactly 120 parallel classes: note that 18 and 120 are the upper bounds (6) and (7) from Theorem 2.3 respectively. In addition, the 2-rank of the incidence matrix of each partial geometry $p g(14,8,7)$ is equal to the 2-rank of the underlying projective plane.

Table 7 contains data about the partial geometries $p g(8,14,7)$ arising from maximal arcs of degree 8 .

Note 2 An interesting open problem is to find an analogue of Mathon's partial geometry when $q=16$, that is, a partial geometry $p g(14,8,7)$ that does not arises from any hyperoval in a projective plane of order 16 , or equivalently, according to Theorem 2.4, its dual geometry does not contain a set of 120 pairwise orthogonal parallel classes of lines. The line graph of such a partial geometry has to be isomorphic to $\bar{T}(18)$, and every line corresponds to a clique of size nine of $\bar{T}(18)$. Since the graph $\bar{T}(18)$ contains

$$
\frac{18 !}{2^{9} 9 !}=34,459,425
$$

cliques of size 9 , finding a collection of 255 cliques of size 9 , every two sharing at most one vertex, would be a very challenging computational problem, at the least.

If $d=4$, the only projective plane of order 16 for which all maximal arcs of degree 4 have been classified up to equivalence, is the Desarguesian plane $P G(2,16)$ (Ball and Blokhuis [1]). The maximal arcs of degree 4 have not

\footnotetext{
${ }^{2}$ Two hyperovals, or more generally, two maximal arcs in a projective plane $\mathcal{P}$ are equivalent if one can be obtained from the other by applying an automorphism of $\mathcal{P}$.
} 
been classified completely in any of the known non-Desarguesian planes of order 16, although such arcs have been found in all but four of the known nonDesarguesian planes of order 16 [9], [10], 16]. All currently known maximal arcs of degree 4 are available online at

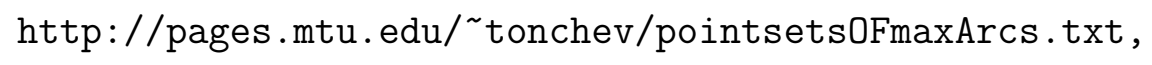

where the maximal arcs are listed as sets of points in the the projective planes of order 16 that are available online at

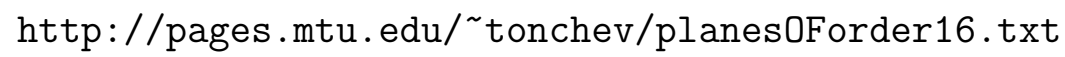

Using Magma [5], we classified, up to isomorphism, the partial geometries $p g(12,12,9)$ arising from the known maximal arcs of degree 4 in projective planes of order 16, and computed the 2-rank of their incidence matrices and all parallel classes of lines. Table 8 summarizes the data about these partial geometries. Up to isomorphism, there are 59 such geometries (13 self-dual and 23 non-self-dual ones). Similarly to the previous cases for degrees other than 4 , or planes of smaller order, the 2-rank of each partial geometry turned out to be equal to the 2-rank of the corresponding plane, and the total number of parallel classes always matches the upper bound of Theorem 2.3.

These observations motivate us to formulate the following conjectures.

Conjecture 4.1 Let $G$ be a partial geometry with parameters (4) for some integer numbers $d \geq 2, d^{\prime} \geq 2$. Then either $G$ does not arise from a maximal arc of degree $d$ in a projective plane of order $q=d d^{\prime}$, or $G$ is obtainable from exactly one projective plane of order $q=d d^{\prime}$ via Construction [2.1.

Conjecture 4.2 If a partial geometry $G$ with parameters (4) for some $d=$ $2^{i} \geq 2, d^{\prime}=2^{j} \geq 2$ arises from a maximal arc of degree $d$ in a projective plane $\mathcal{P}$ of order $q=2^{i+j}$ via Construction [2.1, then the 2-rank of the $(0,1)$ incidence matrix of $G$ is equal to the 2-rank of the $(0,1)$-incidence matrix of $\mathcal{P}$.

\section{References}

[1] Ball, S., Blokhuis A.: The classification of maximal arcs in small Desarguesian planes, Bull. Belg. Math. Soc. 9 (2009), 433-445.

[2] Ball, S., Blokhuis A., Mazzocca F.: Maximal arcs in Desarguesian planes of odd order do not exist. Combinatorica 17 (1997), 31-41.

[3] Beth T., Jungnickel D., Lenz, H.: Design Theory, 2nd edition. Cambridge University Press, Cambridge 1999. 
[4] Bose, R. C.: Strongly regular graphs, partial geometries and partially balanced designs, Pacific J. Math. 13, No. 2 (1963), 389 - 419.

[5] Bosma, W., Cannon J.: Handbook of Magma Functions, School of Mathematics and Statistics, University of Sidney, Sidney, July 22 (1999).

[6] Chang, Li-Chien: The uniqueness and non-uniqueness of the triangular association schemes, Science Record, Peking, New Ser. 3 (1959), 604 613.

[7] De Clerk, F.: The pseudo-geometric $(t, s, s-1)$-graphs, Simon Stevin 55 (1979), 301-317.

[8] Denniston R.H.F.: Some maximal arcs in finite projective planes. $J$. Combin. Theory 6 317-319 (1969).

[9] Gezek, M., Mathon, R., Tonchev, V. D.: Maximal arcs, codes, and new links between projective planes, Electronic J. Combinatorics, Volume 27, Issue 1 (2020) Article P1.62, DOI: https://doi.org/10.37236/9008.

[10] Gezek, M., Wagner, T., Tonchev, V. D.: Maximal arcs in projective planes of order 16 and related designs, Advances in Geometry, 19 (3) (2019), $345-351$.

[11] Hamilton, N.: Some maximal arcs in Hall planes, J. Geom. 52 (1995), 101-107.

[12] Hamilton, N.: Some inherited maximal arcs in derived dual dual translation planes, Geom. Dedicata 55 (1995), $165-173$.

[13] Hamilton, N.: Some maximal arcs in derived dual Hall planes, European J. Combin. 15 (1994), $525-532$.

[14] Hamilton, N., Mathon, R.: More maximal arcs in Desarguesian projective planes and their geometric structure,Adv. Geom. 3 (2003), $251-$ 261.

[15] Hamilton, N., Mathon, R.: On the spectrum of non-Denniston maximal arcs in $P G\left(2,2^{h}\right)$, European J. Combin. 25 (2004), 415 - 421.

[16] Hamilton N., Stoichev S. D., Tonchev V. D.: Maximal arcs and disjoint maximal arcs in projective planes of order 16. J. Geometry 67 (2000), 117-126.

[17] Hoffman A. J.: On the uniqueness of the triangular association scheme, Ann. Math. Statist. 31 (1960), 492 - 497. 
[18] Hoffman, A. J.: On eigenvalues and colorings of graphs, in: Graph theory and its applications (B. Harris, ed.), pp. 79-91, Academic Press, New York, 1970.

[19] Lam, C.W.H., Thiel, L., Swiercz, S., and McKay, J.: The nonexistence of ovals in a projective plane of order 10, Discr. Math. 45 (1983), 319 321.

[20] Lunelli L., Sce M.: K-archi completi nei piani proiettivi desarguesiani di rango 8 e 16. Centro di Calcoli Numerici, Politecnico di Milano, Milan 1958, pp. 15.

[21] Mathon, R.: The partial geometries pg $(5,7,3)$, Congress. Numer. 31 (1981), 129-139.

[22] Mathon, R.: New maximal arcs in Desarguesian planes, J. Combin. Theory Ser. A 97 (2002), $353-368$.

[23] Moorhouse, E. G.: Projective Planes of Order 16. http://www.uwyo.edu/moorhouse/pub/planes16/.

[24] Niskanen, S., Östergård, P. R. J.: Cliquer User's Guide, Version 1.0. Tech. Rep. T48, Communications Laboratory, Helsinki University of Technology, Espoo, Finland, 2003.

[25] Payne S.E. and Thas, J. A.: Generalized Quadrangles, Pitman, New York, 1985.

[26] Penttila, T., Royle, G. F., Simpson, M. K.:, Hyperovals in the known projective planes of order 16. J. Combin. Designs 4 (1996), 59 - 65.

[27] Reichard S. and Woldar A.: Constructing partial geometries from overlarge sets of Steiner systems, preprint, Research Gate, July 2019, DOI: 10.13140/RG.2.2.16055.37289.

[28] Thas, J. A.: Construction of partial geometries, Simon Stevin 46 (1973), 95-98.

[29] Thas, J. A.: Construction of maximal arcs and partial geometries, Geom. Dedicata 3 (1974), 61-64.

[30] Thas, J. A.: Construction of maximal arcs and dual ovals in translation planes, European J. Combin. 1 (1980), 189-192.

[31] Thas, J. A.: Partial Geometries, in: Handbook of Combinatorial Designs, C. J. Colbourn and J. F. Dinitz, eds., Chapman \& Hall/CRC, 2007, pp. $557-561$. 
[32] Tonchev, V. D.: On resolvable Steiner 2-designs and maximal arcs in projective planes. Designs, Codes, and Cryptography 84 (2017), 165 172.

[33] V.D. Tonchev: Combinatorial Configurations, Longman- Wiley, New York (1988).

[34] Wallis, W. D.: Configurations arising from maximal arcs, J. Combin. Theory A 15 (1973), 115-119. 


\section{Appendix}

\begin{tabular}{|c|c|c|c|c|}
\hline No. & Hyperoval & $|A u t(G)|$ & 2-rank & \# Par. cl. \\
\hline 1 & PG(2,16)hyp.1 & 144 & 82 & 120 \\
\hline 2 & PG(2,16)hyp.2 & 16320 & 82 & 120 \\
\hline 3 & DEMPhyp.1 & 16 & 102 & 120 \\
\hline 4 & DEMPhyp.2 & 16 & 101 & 120 \\
\hline 5 & DEMPhyp.3 & 16 & 102 & 120 \\
\hline 6 & DEMPhyp.4 & 16 & 102 & 120 \\
\hline 7 & DEMPhyp.5 & 16 & 100 & 120 \\
\hline 8 & DEMPhyp.6 & 16 & 102 & 120 \\
\hline 9 & DEMPhyp.7 & 16 & 101 & 120 \\
\hline 10 & DEMPhyp.8 & 16 & 102 & 120 \\
\hline 11 & DEMPhyp.9 & 16 & 101 & 120 \\
\hline 12 & DEMPhyp.10 & 16 & 102 & 120 \\
\hline 13 & DEMPhyp.11 & 16 & 101 & 120 \\
\hline 14 & DEMPhyp.12 & 64 & 100 & 120 \\
\hline 15 & DEMPhyp.13 & 64 & 100 & 120 \\
\hline 16 & DEMPhyp.14 & 64 & 100 & 120 \\
\hline 17 & DEMPhyp.15 & 80 & 102 & 120 \\
\hline 18 & dDEMPhyp.1 & 2 & 102 & 120 \\
\hline 19 & dDEMPhyp.2 & 6 & 102 & 120 \\
\hline 20 & SEMI4hyp.1 & 16 & 98 & 120 \\
\hline 21 & SEMI4hyp.2 & 16 & 98 & 120 \\
\hline 22 & SEMI4hyp.3 & 16 & 98 & 120 \\
\hline 23 & SEMI2hyp.1 & 3 & 98 & 120 \\
\hline 24 & SEMI2hyp.2 & 8 & 98 & 120 \\
\hline 25 & SEMI2hyp.3 & 8 & 98 & 120 \\
\hline 26 & SEMI2hyp.4 & 16 & 98 & 120 \\
\hline 27 & SEMI2hyp.5 & 16 & 98 & 120 \\
\hline 28 & SEMI2hyp.6 & 16 & 97 & 120 \\
\hline 29 & SEMI2hyp.7 & 16 & 98 & 120 \\
\hline 30 & SEMI2hyp.8 & 16 & 97 & 120 \\
\hline 31 & SEMI2hyp.9 & 16 & 98 & 120 \\
\hline 32 & SEMI2hyp.10 & 16 & 97 & 120 \\
\hline 33 & SEMI2hyp.11 & 16 & 98 & 120 \\
\hline 34 & SEMI2hyp.12 & 16 & 98 & 120 \\
\hline 35 & SEMI2hyp.13 & 16 & 97 & 120 \\
\hline 36 & SEMI2hyp.14 & 26 & 98 & 120 \\
\hline 37 & SEMI2hyp.15 & 16 & 98 & 120 \\
\hline 38 & SEMI2hyp.16 & 32 & 98 & 120 \\
\hline 39 & SEMI2hyp.17 & 32 & 97 & 120 \\
\hline 40 & LMRHhyp.1 & 16 & 100 & 120 \\
\hline 41 & LMRHhyp.2 & 16 & 103 & 120 \\
\hline 42 & LMRHhyp.3 & 16 & 103 & 120 \\
\hline 43 & LMRHhyp.4 & 16 & 103 & 120 \\
\hline 44 & LMRHhyp.5 & 64 & 103 & 120 \\
\hline 45 & LMRHhyp.6 & 112 & 99 & 120 \\
\hline 46 & dLMRHhyp.1 & 14 & 105 & 120 \\
\hline
\end{tabular}

Table 7: The $\operatorname{pg}(8,14,7)$ 's arising from maximal (120,8)-arcs 


\begin{tabular}{|c|c|c|c|c|}
\hline No. & Hyperoval & $|A u t(G)|$ & 2-rank & \# Par. cl. \\
\hline 47 & MATHhyp.1 & 8 & 107 & 120 \\
\hline 48 & dMATHhyp.1 & 4 & 108 & 120 \\
\hline 49 & dMATHhyp.2 & 4 & 108 & 120 \\
\hline 50 & dMATHhyp.3 & 8 & 109 & 120 \\
\hline 51 & HALLhyp.1 & 16 & 97 & 120 \\
\hline 52 & HALLhyp.2 & 64 & 98 & 120 \\
\hline 53 & HALLhyp.3 & 64 & 97 & 120 \\
\hline 54 & HALLhyp.4 & 320 & 97 & 120 \\
\hline 55 & dHALLhyp.1 & 2 & 98 & 120 \\
\hline 56 & dHALLhyp.2 & 2 & 98 & 120 \\
\hline 57 & dHALLhyp.3 & 6 & 98 & 120 \\
\hline 58 & BBH1hyp.1 & 8 & 107 & 120 \\
\hline 59 & BBH1hyp.2 & 16 & 109 & 120 \\
\hline 60 & BBH1hyp.3 & 32 & 107 & 120 \\
\hline 61 & JOWKhyp.1 & 16 & 99 & 120 \\
\hline 62 & JOWKhyp.2 & 16 & 99 & 120 \\
\hline 63 & JOWKhyp.3 & 16 & 100 & 120 \\
\hline 64 & JOWKhyp.4 & 16 & 99 & 120 \\
\hline 65 & JOWKhyp.5 & 64 & 99 & 120 \\
\hline 66 & JOWKhyp.6 & 112 & 99 & 120 \\
\hline 67 & dJOWKhyp.1 & 14 & 99 & 120 \\
\hline 68 & JOHNhyp.1 & 16 & 111 & 120 \\
\hline 69 & DSFPhyp.1 & 16 & 103 & 120 \\
\hline 70 & DSFPhyp.2 & 16 & 103 & 120 \\
\hline 71 & DSFPhyp.3 & 16 & 103 & 120 \\
\hline 72 & DSFPhyp.4 & 16 & 103 & 120 \\
\hline 73 & DSFPhyp.5 & 16 & 103 & 120 \\
\hline 74 & DSFPhyp.6 & 16 & 103 & 120 \\
\hline 75 & DSFPhyp.7 & 16 & 103 & 120 \\
\hline 76 & DSFPhyp.8 & 16 & 103 & 120 \\
\hline 77 & DSFPhyp.9 & 16 & 103 & 120 \\
\hline 78 & DSFPhyp.10 & 16 & 103 & 120 \\
\hline 79 & DSFPhyp.11 & 16 & 103 & 120 \\
\hline 80 & DSFPhyp.12 & 16 & 103 & 120 \\
\hline 81 & DSFPhyp.13 & 16 & 103 & 120 \\
\hline 82 & DSFPhyp.14 & 16 & 103 & 120 \\
\hline 83 & DSFPhyp.15 & 16 & 103 & 120 \\
\hline 84 & DSFPhyp.16 & 16 & 102 & 120 \\
\hline 85 & DSFPhyp.17 & 16 & 103 & 120 \\
\hline 86 & DSFPhyp.18 & 16 & 103 & 120 \\
\hline 87 & DSFPhyp.19 & 16 & 103 & 120 \\
\hline 88 & DSFPhyp.20 & 16 & 103 & 120 \\
\hline 89 & DSFPhyp.21 & 16 & 103 & 120 \\
\hline 90 & DSFPhyp.22 & 64 & 103 & 120 \\
\hline 91 & BBH2hyp.1 & 4 & 111 & 120 \\
\hline 93 & BBH2hyp.2 & 4 & 111 & 120 \\
\hline & BBS4hyp.1 & 16 & 111 & 120 \\
\hline
\end{tabular}

Table 7: The $\operatorname{pg}(8,14,7)$ 's arising from maximal (120,8)-arcs (continued). 


\begin{tabular}{|c|c|c|c|c|c|c|}
\hline$\#$ & $\begin{array}{c}\text { Maximal } \\
52 \text {-arc }\end{array}$ & $|A u t(G)|$ & 2 -rank & $\begin{array}{c}\# \\
\text { of } \\
\text { par. clas. }\end{array}$ & $\begin{array}{c}\text { Isomorphic } \\
\text { to } \\
\text { its dual? }\end{array}$ & $\begin{array}{c}\text { Isomorphic } \\
\text { to } \\
\text { others? }\end{array}$ \\
\hline 1 & PG(2,16).1 & 68 & 82 & 52 & Yes & No \\
\hline 2 & PG(2,16).2 & 408 & 82 & 52 & Yes & No \\
\hline 3 & DEMP.1 & 24 & 102 & 52 & No & No \\
\hline 4 & DEMP.2 & 144 & 102 & 52 & No & No \\
\hline 5 & DEMP.3 & 24 & 102 & 52 & No & No \\
\hline 6 & DEMP.4 & 48 & 102 & 52 & No & No \\
\hline 7 & DEMP.5 & 4 & 102 & 52 & No & No \\
\hline 8 & SEMI4.1 & 96 & 98 & 52 & Yes & No \\
\hline 9 & SEMI2.1 & 24 & 98 & 52 & Yes & No \\
\hline 10 & SEMI2.2 & 144 & 98 & 52 & Yes & No \\
\hline 11 & SEMI2.3 & 32 & 98 & 52 & Yes & No \\
\hline 12 & SEMI2.4 & 32 & 98 & 52 & Yes & No \\
\hline 13 & SEMI2.5 & 16 & 98 & 52 & Yes & No \\
\hline 14 & SEMI2.6 & 48 & 98 & 52 & No & SEMI2.7 \\
\hline 15 & SEMI2.7 & 48 & 98 & 52 & No & SEMI2.6 \\
\hline 16 & LMRH.1 & 96 & 106 & 52 & No & No \\
\hline 17 & LMRH.2 & 32 & 106 & 52 & No & No \\
\hline 18 & MATH.1 & 24 & 109 & 52 & No & No \\
\hline 19 & MATH.2 & 32 & 108 & 52 & No & No \\
\hline 20 & MATH.3 & 32 & 108 & 52 & No & No \\
\hline 21 & MATH.4 & 32 & 108 & 52 & No & No \\
\hline 22 & MATH.5 & 16 & 109 & 52 & No & No \\
\hline 23 & MATH.6 & 16 & 109 & 52 & No & No \\
\hline 24 & MATH.7 & 16 & 109 & 52 & No & No \\
\hline 25 & HALL.1 & 24 & 98 & 52 & No & No \\
\hline 26 & HALL.2 & 4 & 98 & 52 & No & No \\
\hline 27 & BBH1.1 & 24 & 110 & 52 & Yes & No \\
\hline 28 & BBH1.2 & 32 & 110 & 52 & Yes & No \\
\hline 29 & BBH1.3 & 4 & 110 & 52 & Yes & No \\
\hline 30 & JOWK.1 & 16 & 100 & 52 & No & No \\
\hline 31 & JOWK.2 & 32 & 100 & 52 & No & No \\
\hline 32 & JOHN.1 & 32 & 113 & 52 & No & No \\
\hline 33 & JOHN.2 & 32 & 113 & 52 & No & No \\
\hline 34 & JOHN.3 & 32 & 113 & 52 & No & No \\
\hline 35 & JOHN.4 & 32 & 113 & 52 & No & No \\
\hline 36 & DSFP.1 & 24 & 106 & 52 & No & No \\
\hline & & & & & & \\
\hline
\end{tabular}

Table 8: The $\operatorname{pg}(12,12,9)$ 's arising from maximal $(52,4)$-arcs 